International Journal of Pure and Applied Mathematics

Volume 102 No. 4 2015, 605-611

ISSN: 1311-8080 (printed version); ISSN: 1314-3395 (on-line version)

url: http://www.ijpam.eu

doi: http://dx.doi.org/10.12732/ijpam.v102i4.2

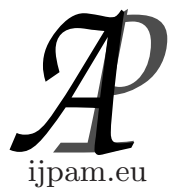

\title{
HAMILTON-CONNECTIVITY IN \\ BALANCED BIPARTITE GRAPHS
}

\author{
Yusleidy Alcalá ${ }^{\S}$, Daniel Brito ${ }^{2}$, Oscar Castro ${ }^{3}$, Lope Marín ${ }^{4}$ \\ 1,2,3,4 Departmento de Matemáticas \\ Escuela de Ciencias \\ Núcleo de Sucre \\ Universidad de Oriente \\ Cumaná 6101-A, Apartado 245, VENEZUELA
}

\begin{abstract}
Let $G$ be a balanced bipartite graph of order $2 n$ and minimum degree $\delta(G) \geq 4$. If for every balanced independent set $S$ of four vertices $|N(S)| \geq n+2$, then $G$ is Hamiltonian connected. This is an improvement of the bound given by [4].
\end{abstract}

AMS Subject Classification: 05C38, 05C45, 05C70

Key Words: hamiltonian connected, balanced bipartite graph, neighborhood union

\section{Introduction}

We use [2] for terminology and notation not defined here. We denote by $V(G)$ and $E(G)$ the vertex set and the edge set of a simple, finite and undirected graph $G$. According to the (arbitrary) orientation of a cycle $C$ of $G$, the successor and predecessor of a vertex $z$ of $C$ are denoted by $z^{+}$and $z^{-}$, respectively. Let $G=(A, B, E)$ be a balanced bipartite simple graph of order $2 n$, i.e. a graph with a bipartition into two independent vertex sets of the same cardinality.

Received: October 3, 2014

(C) 2015 Academic Publications, Ltd.

$\S$ Correspondence author url: www.acadpubl.eu 
$N(S)$ is the neighborhood union of a balanced independent set $S$ of four vertices, i.e. an independent set containing two vertices from each side of the bipartition. $G$ is Hamiltonian connected if for every two vertices one on each side of the bipartition there exists a Hamiltonian path connecting them.

A 2-dumbbell, is be a graph that consists of two disjoint cycles $C_{1}$ and $C_{2}$ which are joined by two disjoint paths $P_{1}$ and $P_{2}$ with $V\left(C_{i}\right) \cap V\left(P_{i}\right)=\phi$ such that the neighbors of the extremes of $P_{1}$ in $C_{i},(i=1,2)$, are consecutive with the neighbors of the extremes of $P_{2}$ in $C_{i}$.

The investigation of certain extremal problems involving neighborhood union conditions for balanced independent sets of cardinality four was initiated by Amar et al. [1]. They posed the following question: Let $H_{14}$ denote the class of graphs obtained from the graph depicted in Fig 1, where some (or all) of the four possible edges joining the top to the bottom might be present as well.

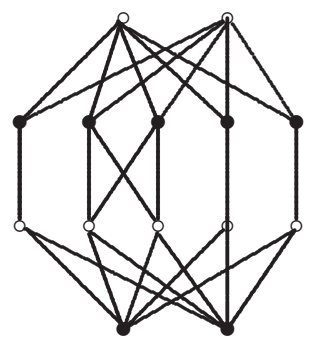

Figure 1: The minimal graph of $H_{14}$

Conjeture 1.1. (Amar et al. [1]). Let $G=(A, B, E)$ be a balanced bipartite graph of order $2 n$ with $\delta(G) \geq 3$. If for every balanced independent set $S$ with $|S|=4$, we have $|N(S)|>n$, then $G$ is Hamiltonian or $G \in H_{14}$.

They also proved the following:

Theorem 1.2 (Amar et al. [1]). Let $G=(A, B, E)$ be a balanced bipartite graph of order $2 n$ with $\delta(G) \geq 3$. If for every balanced independent set $S$ with $|S|=4$, we have $|N(S)|>n+2$, then $G$ is Hamiltonian.

Since then, the following results have been obtained: 
Theorem 1.3 (Brito y Lárez. [3]). Let $G=(A, B, E)$ be a balanced bipartite graph of order $2 n$ and minimum degree $\delta(G) \geq 4$. If for every balanced independent set $S$ with $|S|=4$, we have $|N(S)|>n$, then $G$ is Hamiltonian.

Theorem 1.4 (Brito et al. [4]). Let $G=(A, B, E)$ a balanced bipartite graph of order $2 n$ and minimum degree $\delta(G) \geq 3$. If for every balanced independent set $S$ with $|S|=4$,we have $|N(S)|>n+2$, then $G$ is Hamiltonian connected.

\section{Previous Lemma}

In the proofs the following subgraph, called $1-2$ dumbbell,the graph depicted in Fig 2, is defined as a dumbbell in which one of the extremes of each path are adjacent. Furthermore the neighbors of the ends of the paths consecutive in the cycle are adjacent to distance one-neighbors of the ends of the other path.

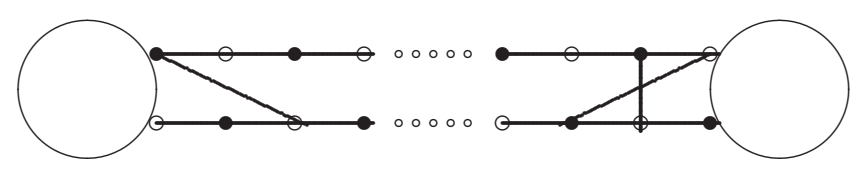

Figure 2: The 1- 2-dumbbell

Lemma 2.1 Let $G=(A, B, E)$ be a balanced bipartite graph of order $2 n$ and minimum degree $\delta(G) \geq 4, C=a_{1} b_{1} a_{2} b_{2} \ldots a_{n} b_{n} a_{1}$ a hamiltonian cycle of $G$ and $D$, a maximal spanning $1-2$ dumbbell of $G$. If $G$ does not have a $u v$-hamiltonian path with $u=b_{n}$ and $v=a_{j}$, then:

1) A balanced independent set $S$ exists such that $|S|=4$.

2) $N^{-}(S \cap A) \cap N(S \cap B)=\emptyset$.

Proof. Let $G=(A, B, E)$ be a balanced bipartite graph of order $2 n$ and minimum degree $\delta(G) \geq 4, C=a_{1} b_{1} a_{2} b_{2} \ldots a_{n} b_{n} a_{1}$, a hamiltonian cycle of 
$G$ and $D$, a maximal spanning $1-2$ dumbbell of $G$, with an orientation $a_{1} C_{1}^{+} b_{s} P_{1} a_{t} C_{2}^{+} b_{j} P_{2} a_{1}$.

By definition of $1-2$ dumbbell let $a_{1}$ be one vertex in the cycle $C_{1}$ neighbor of one end of the path $P_{2}$ and $b_{j}$ one vertex in the cycle $C_{2}$ neighbor of one end of the path $P_{2}$ and similarly let $b_{s}$ one vertex in the cycle $C_{1}$ neighbor of one end of the path $P_{1}$ and $a_{t}$ one vertex in the cycle $C_{2}$ neighbor of one end of the path $P_{1}$, such that $a_{1}\left(a_{t}\right)$ and $b_{s}\left(b_{j}\right)$ are neighbor consecutive in $C_{1}\left(C_{2}\right)$, respectively.There are also sides $a_{1} b_{s}^{++}, b_{j} a_{t}^{--}, b_{j}^{+} a_{t}^{-}$. If $G$ does not have a $u v$-hamiltonian path with $u=b_{n}$ and $v=a_{j}$ then:

i) $a_{1} b_{j} \notin E(G)$; for otherwise $b_{n} P_{2}^{-} b_{j}^{+} b_{j} a_{1} C_{1}^{+} b_{s} b_{s}^{+} P_{1}^{+} a_{t}^{-} a_{t} C_{2}^{+} a_{j}$ is a $u v$ hamiltonian path with $u=b_{n}$ and $v=a_{j}$.

ii) $a_{1} a_{t}^{-} \notin E(G)$; for otherwise $b_{n} P_{2}^{-} b_{j}^{+} b_{j} a_{t}^{--} P_{1}^{-} b_{s}^{+} b_{s} C_{1}^{-} a_{1} a_{t}^{-} a_{t} C_{2}^{+} a_{j}$ is a $u v$-hamiltonian path with $u=b_{n}$ and $v=a_{j}$.

iii) $b_{s}^{+} b_{j} \notin E(G)$; for otherwise $b_{n} P_{2}^{-} b_{j}^{+} b_{j} b_{s}^{+} b_{s} C_{1}^{-} a_{1} b_{s}^{++} P_{1}^{+} a_{t}^{-} a_{t} C_{2}^{+} a_{j}$ is a $u v$-hamiltonian path with $u=b_{n}$ and $v=a_{j}$.

iv) $b_{s}^{+} a_{t}^{-} \notin E(G)$; for otherwise $b_{n} P_{2}^{-} b_{j}^{+} b_{j} a_{t}^{--} P_{1}^{-} b_{s}^{++} a_{1} C_{1}^{+} b_{s} b_{s}^{+} a_{t}^{-} a_{t} C_{2}^{+} a_{j}$ is a $u v$-hamiltonian path with $u=b_{n}$ and $v=a_{j}$.

Then there exists a balanced independent set, $S$, of cardinality 4 , with $S=\left\{a_{1}, b_{j}, b_{s}^{+}, a_{t}^{-}\right\}$

Let's assume otherwise in 2; i.e., $\left(N^{-}(S \cap A) \cap N(S \cap B)\right) \neq \emptyset$. Let, without loss of generality, $S=\left\{a_{1}, b_{j}, b_{s}^{+}, a_{t}^{-}\right\}$con $a_{1}, b_{s}^{+} \in A$ y $b_{j}, a_{t}^{-} \in B$

Let, $a_{k} \in\left(N^{-}(S \cap A) \cap N(S \cap B)\right)$, such that $a_{k} \in\left(N^{-}\left(a_{1}, b_{s}^{+}\right)\right)$and $a_{k} \in\left(N\left(b_{j}, a_{t}^{-}\right)\right)$by definition $a_{k}^{+} a_{1} \in E(G)$ o $a_{k}^{+} b_{s}^{+} \in E(G)$ and $a_{k} b_{j} \in E(G)$ о $a_{k} a_{t}^{-} \in E(G)$.

Consider the following cases:

i) $1<k<s<t<j<n$

If $a_{k}^{+} b_{s}^{+} \in E(G)$ and $a_{k} b_{j} \in E(G)$; then

$$
b_{n} P_{2}^{-} b_{j} a_{k} C_{1}^{-} a_{k}^{+} b_{s}^{+} P_{1}^{+} a_{t}^{-} a_{t} C_{2}^{+} a_{j}
$$

is a $u v$-hamiltonian path with $u=b_{n}$ and $v=a_{j}$.

If $a_{k}^{+} b_{s}^{+} \in E(G)$ and $a_{k} a_{t}-\in E(G)$; then

$$
b_{n} P_{2}^{-} b_{j} a_{t}^{--} P_{1}^{-} b_{s}^{+} a_{k}^{+} C_{1}^{+} a_{k} a_{t}^{-} a_{t} C_{2}^{+} a_{j}
$$

is a $u v$-hamiltonian path with $u=b_{n}$ and $v=a_{j}$. 
ii) $1<s<k<t<j<n 1$

If $a_{k}^{+} a_{1} \in E(G)$ and $a_{k} b_{j} \in E(G)$; then

$$
b_{n} P_{2}^{-} b_{j} a_{k} P_{1}^{-} b_{s}^{+} b_{s} C_{1}^{-} a_{1} a_{k}^{+} P_{1}^{+} a_{t}^{-} a_{t} C_{2}^{+} a_{j}
$$

is a $u v$-hamiltonian path with $u=b_{n}$ and $v=a_{j}$.

If $a_{k}^{+} a_{1} \in E(G)$ and $a_{k} a_{t}-\in E(G)$; then

$$
b_{n} P_{2}^{-} b_{j} a_{t}^{--} P_{1}^{-} a_{k}^{+} a_{1} C_{1}^{+} b_{s} b_{s}^{+} P_{1}^{+} a_{k} a_{t}^{-} a_{t} C_{2}^{+} a_{j}
$$

is a $u v$-hamiltonian path with $u=b_{n}$ and $v=a_{j}$.

If $a_{k}^{+} b_{s}^{+} \in E(G)$ and $a_{k} b_{j} \in E(G)$; then

$$
b_{n} P_{2}^{-} b_{j} a_{k} P_{1}^{-} b_{s}^{++} a_{1} C_{1}^{+} b_{s} b_{s}^{+} a_{k}^{+} P_{1}^{+} a_{t}^{-} a_{t} C_{2}^{+} a_{j}
$$

is a $u v$-hamiltonian path with $u=b_{n}$ and $v=a_{j}$.

If $a_{k}^{+} b_{s}^{+} \in E(G)$ and $a_{k} a_{t}^{-} \in E(G)$; then

$$
b_{n} P_{2}^{-} b_{j} a_{t}^{--} P_{1}^{-} a_{k}^{+} b_{s}^{+} b_{s} C_{1}^{-} a_{1} b_{s}^{++} P_{1}^{+} a_{k} a_{t}^{-} a_{t} C_{2}^{+} a_{j}
$$

is a $u v$-hamiltonian path with $u=b_{n}$ and $v=a_{j}$.

iii) $1<s<t<k<j<n$.

If $a_{k}^{+} a_{1} \in E(G)$ and $a_{k} a_{t}^{-} \in E(G)$; then

$$
b_{n} P_{2}^{-} b_{j} a_{t} C_{2}^{+} a_{k} a_{t}^{-} P_{1}^{-} b_{s}^{+} b_{s} C_{1}^{-} a_{1} a_{k}^{+} C_{2}^{+} a_{j}
$$

is a $u v$-hamiltonian path with $u=b_{n}$ and $v=a_{j}$.

If $a_{k}^{+} b_{s}^{+} \in E(G)$ and $a_{k} a_{t}^{-} \in E(G)$; then

$$
b_{n} P_{2}^{-} b_{j} a_{t} C_{2}^{+} a_{k} a_{t}^{-} P_{1}^{-} b_{s}^{++} a_{1} C_{1}^{+} b_{s} b_{s}^{+} a_{k}^{+} C_{2}^{+} a_{j}
$$

is a $u v$-hamiltonian path with $u=b_{n}$ and $v=a_{j}$.

iv) $1<s<t<j<k<n$. 
If $a_{k}^{+} a_{1} \in E(G)$ and $a_{k}^{-} b_{j} \in E(G)$; then

$$
b_{n} P_{2}^{-} a_{k}^{+} a_{1} C_{1}^{+} b_{s} b_{s}^{+} P_{1}^{+} a_{t}^{--} b_{j} a_{k}^{-} P_{2}^{-} b_{j}^{+} a_{t}^{-} a_{t} C_{2}^{+} a_{j}
$$

is a $u v$-hamiltonian path with $u=b_{n}$ and $v=a_{j}$.

If $a_{1} a_{k}^{+} \in E(G)$ and $a_{k} a_{t}^{-} \in E(G)$; then

$$
b_{n} P_{2}^{-} a_{k}^{+} a_{1} C_{1}^{+} b_{s} b_{s}^{+} P_{1}^{+} a_{t}^{-} a_{k} P_{2}^{-} b_{j} a_{t} C_{2}^{+} a_{j}
$$

is a $u v$-hamiltonian path with $u=b_{n}$ and $v=a_{j}$.

If $a_{k}^{+} b_{s}^{+} \in E(G)$ and $a_{k} b_{j} \in E(G)$; then

$$
b_{n} P_{2}^{-} a_{k}^{+} b_{s}^{+} b_{s} C_{1}^{-} a_{1} b_{s}^{++} P_{1}^{+} a_{t}^{-} b_{j}^{+} P_{2}^{+} a_{k} b_{j} a_{t} C_{2}^{+} a_{j}
$$

is a $u v$-hamiltonian path with $u=b_{n}$ and $v=a_{j}$.

If $a_{k}^{+} b_{s}^{+} \in E(G)$ and $a_{k} a_{t}^{-} \in E(G)$; then

$$
b_{n} P_{2}^{-} a_{k}^{+} b_{s}^{+} b_{s} C_{1}^{-} a_{1} b_{s}^{++} P_{1}^{+} a_{t}^{-} a_{k} P_{2}^{-} b_{j} a_{t} C_{2}^{+} a_{j}
$$

is a $u v$-hamiltonian path with $u=b_{n}$ and $v=a_{j}$.

\section{Main Result}

In this paper we show the following theorem:

Theorem 3.1 Let $G=(A, B, E)$ a balanced bipartite graph of order $2 n$ and minimum degree $\delta(G) \geq 4$. If for every balanced independent set $S$ with $|S|=4$, we have $|N(S)| \geq n+2$, then $G$ is Hamiltonian connected.

Proof. Let $G=(A, B, E)$ be a balanced bipartite graph of order $2 n$ and minimum degree $\delta(G) \geq 4, C=a_{1} b_{1} a_{2} b_{2} \ldots a_{n} b_{n} a_{1}$ a hamiltonian cycle of $G$ and $D$, a maximal spanning $1-2$ dumbbell of $G$, with an orientation $a_{1} C_{1}^{+} b_{s} P_{2} a_{t} C_{2}^{+} b_{j} P_{1} a_{1}$ By definition of $1-2$ dumbbell let $a_{1}$ be one vertex in the cycle $C_{1}$ neighbor of one end of the path $P_{1}$ and $b_{j}$ one vertex in the cycle $C_{2}$ neighbor of one end of the path $P_{1}$ and similarly let $b_{s}$ be one vertex in the cycle $C_{1}$ neighbors of one end of the path $P_{2}$ and $a_{t}$ one vertex in the cycle 
$C_{2}$ neighbor of one end of the path $P_{2}$, such that $a_{1}\left(a_{t}\right)$ and $b_{s}\left(b_{j}\right)$ are neighbor consecutive in $C_{1}\left(C_{2}\right)$, respectively.There are also sides $a_{1} b_{s}^{++}, b_{j} a_{t}^{--}, b_{j}^{+} a_{t}^{-}$.

Suppose that $G$ does not have a $u v$-Hamiltonian path with $u=b_{n}$ and $v=a_{j}$.

Let, without loss of generality, $a_{1}, b_{s}^{+}$be in $A$ and $b_{j}, a_{t}^{-}$in $B$.

Let $S=\left\{a_{1}, b_{j}, b_{s}^{+}, a_{t}^{-}\right\}$be the independent set with $N\left(a_{1}, b_{s}^{+}\right) \cap N^{+}\left(b_{j}, a_{t}^{-}\right)=$ $\emptyset$, given by Lemma 2.1 .

Then,

$$
\begin{aligned}
n+2 \leq|N(S)| & =\left|N\left(a_{1}, b_{s}^{+}\right)\right|+\left|N\left(b_{j}, a_{t}^{-}\right)\right| \\
& =\left|N^{-}\left(a_{1}, b_{s}^{+}\right)\right|+\left|b_{n}\right|+\left|N\left(b_{j}, a_{t}^{-}\right)\right| \\
& \leq|| N^{-}\left(a_{1}, b_{s}^{+}\right)|\cup| N\left(b_{j}, a_{t}^{-}\right)||+1 \\
& \leq|A|+1 \leq n+1,
\end{aligned}
$$

which contradicts the hypothesis of the theorem.

This proves the theorem.

As an immediate consequence we have

Corollary 3.1. Let $G$ be a balanced bipartite graph of order $2 n$ and minimum degree $\delta(G) \geq 4$. If for every four independent balanced vertices the sum of their degrees is at least $n+2$, then $G$ is hamiltonian connected.

\section{References}

[1] D. Amar, S. Brandt, D. Brito and O. Ordaz, Neighborhood conditions for balanced independent sets in bipartite graphs, Discrete Mathematics, 181 (1998) 31-36.

[2] J. Bondy and U. Murty, Graphs Theory with Applications, North Holland New York, USA (1976).

[3] D. Brito, G. Lárez, Neighborhood conditions for balanced bipartite graphs to be Hamiltonian, International Journal of Pure and Applied Mathematics, 34 (2007), 509-512.

[4] D. Brito, P. Mago and L. Marín, Neighborhood conditions for balanced bipartite graphs to be Hamiltonian connected. International Mathematical Forum, 26 (2010), 1291-1295. 
